\title{
The Role of Technical Quality Control in Histology Laboratories
}

\section{Zuhair M Mohammedsaleh \\ Faculty of Applied Medical Sciences, University Of Tabuk, Tabuk, Kingdom of Saudi Arabia}

*Corresponding author: Zuhair Mohammedsaleh, Faculty of Applied Medical Sciences, University Of Tabuk, Tabuk, Kingdom of Saudi Arabia, Tel: 61399054000 ; Email: zuhair.saleh966@gmail.com

Rec date: Feb 28, 2014, Acc date: May 26, 2014, Pub date: May 28, 2014

Copyright: (c) 2014 Mohammedsaleh ZM. This is an open-access article distributed under the terms of the Creative Commons Attribution License, which permits unrestricted use, distribution, and reproduction in any medium, provided the original author and source are credited.

\begin{abstract}
The importance of laboratories cannot be gainsaid as far as the health of any country is concerned. This is especially considering that laboratories have a bearing on the accuracy of research carried out on varied ailments, as well as the effectiveness of any remedial substance. Of course, there are numerous categories of laboratories with histology laboratories coming as some of the most fundamental. Histology laboratories come with a number of distinctive characteristics that can only be comprehended through comparing the laboratories with other areas of medical laboratories. Nevertheless, they also have to ensure the efficacy and integrity of their results. This underlines the importance of quality control in histology laboratories. Quality control underlines a system pertaining to routine technical activities that are aimed at developing and controlling the quality and accuracy of inventory while it is being developed. This system comes up with consistent and routine checks that identify and address omissions and errors, safeguard the integrity, completeness and correctness of data, while also recording all quality control activities.
\end{abstract}

This Review Article examines the role of quality control in histology laboratories. It examines the varied aspects of quality management including risk identification, risk analysis and risk elimination. On the same note, it examines the convergence between quality management and quality assurance while paying close attention to the role of technical quality control in histology laboratories.

Keywords: Histology; Histopathology; Quality control

\section{Introduction}

Histopathology has been one of the most fundamental sectors or divisions in the biology field in the contemporary human society. It underlines the art of analyzing, as well as interpreting the sizes, shapes and architectural patterns pertaining to tissues and cells in a given specific clinical background, not to mention a science through which that image would be placed in the context of pathobiology knowledge so as to come up with an accurate diagnosis. Underlining the importance histopathology is the fact that it has a bearing on the quality and accuracy of diagnosis. Scholars have noted that all activities and procedures pertaining to histopathology laboratories have to be assessed and monitored in an accurate manner so as to operate safely and effectively. At the heart of histopathologic diagnosis is histology laboratory. As much as histology laboratories are conventionally staffed and managed by experienced and certified technicians and histotechnologists, while pathologists play fundamental role in guiding the manner in which work is carried out, enhancing quality assurance, while ensuring that the final product is up to the set standards.

Histology refers to the study of the anatomy of tissues, cells and organs of plants and animals. The study involves examining organs, tissues and cells by sectioning and staining, and then examining them under a microscope [1]. Histology laboratories come with a number of distinctive characteristics that can only be comprehended through comparing the laboratories with other areas of medical laboratories. Histology laboratories incorporate unique, discrete, irreplaceable, solid and heterogeneous samples, with less than 30 percent of their tasks being automated. In addition, histology laboratories involve over 4500 procedures which are diversified as a result of personal use and preferences. Their results are qualitative and usually difficult to quantify, with batches of work being finished intermittently in a large number of laboratories. On the same note, every step in histology laboratories' work flow necessitates a decision that impacts on the finished slide.

Histology laboratories have a long tradition of internal and external technical quality control. Quality control is a process that ensures certain limits for a product or test have been maintained. It underlines a system pertaining to routine technical activities that are aimed at developing and controlling the quality and accuracy of inventory while it is being developed. This system comes up with consistent and routine checks that identify and address omissions and errors, safeguard the integrity, completeness and correctness of data, while also recording all quality control activities. It is well noted that quality control checks in histopathology laboratories involves accurate identification of patients, fixation, sufficient processing, appropriate techniques for embedding, unacceptable artifacts, microtomy, as well as inspections of controls so as to determine how appropriate or correct immunohistochemical techniques and special stains are. Pathologists are obligated to carry out final quality control examination while reading the slide, as well as determine the adequacy of slides for diagnostic interpretation.

For this reason, quality control has become an essential part of every laboratory's daily operation. The key purpose of this process is to guarantee quality laboratory results by managing all aspects. However, process should not only be applicable on the testing procedure, but it should be applied to ensure quality throughout the total testing 
process, that is, from ordering the test to entering the result on the patient's chart [2].

In histology laboratories, it is not sufficient to have only technical competences, but appropriate medical competence is also required. This helps in quality assurance, because the laboratories results are essential in aiding prescription, diagnosis, and monitoring progress. Furthermore, quality assurance should be achieved because the laboratory results must be reliable, and well documented. This can only be guaranteed the laboratory professions show a high level of ethical responsibility to ensure that the test performed in the lab are of the highest quality [3]. In addition, this can also be achieved by adherence to the technical quality control program.

Active learning is also very important. Histology technicians encounter new information frequently emanating from new discoveries. As such, they should always be ready to learn the new information and its implications in practice, currently and in the future. They should have ability to use instructional tools, methods and procedures appropriately in order to acquire new information [4].

Safety is an equally important aspect of quality control in all histology laboratories. Researchers have established that there are enormous environmental and workplace hazards in histological laboratories. The laboratories are associated with hazardous chemical that may pose a potential risk to workers, as well as, the environment. For this reason, many nations have now passed standards and regulations that are designed to improve working conditions and overcome the potential threats [5]. However, this standards and regulations vary from one country to another, but the underlying theme is universal. Thus, in order to manage risk and improve on safety, medical laboratories and research facilities conform to rules and regulations to regulate hazardsunique to histology. This has resulted to a notable significant improvement in working conditions and quality control.

\section{Quality Management}

Quality assurance is linked with a quality management [6]. In many cases, a quality management system manages to provide the best service for patients and clinicians. In this case, quality is viewed as a measure of how well, a service or product is, and it does the job for which is designed to do. Quality management encompasses with various essentials that together define a comprehensive plan that ensure the quality of test results. These essential addresses the organization need of the test, personnel qualifications and training, method validation, acquisition of reagents and equipment and control over the testing process among others [7]. The internal and external quality management system can attain these essentials by maintaining quality of service, consistency, confidence, continued improvement and standardization. Furthermore, the existence of an appropriate management structure is known to adequately deliver. Laboratory workers are able to work efficiently in their assigned areas and deliver effectively.

The management system should play a significant role in ensuring technical quality control in the laboratory. Since the emergence of accreditation, the management became an important aspect of the daily activities in the histopathology laboratory. The accreditation standards include management as part of the evaluation, and it is, therefore, essential for every laboratory worker to become familiar with the management system and process involved[8]. The management should make sure that quality control correlates with the goals, objectives and policies of the organization. This will ensure that laboratory workers follow the required procedures established by the organization in technical quality control.

Histology technicians should have monitoring skills. They should be able to asses self performance, the performance of workmates and the overall organization [9].

Quality management system involves monitoring and improvement, and is achieved through the process of risk management. In fact, researchers add that laboratory quality control requirements should be based on the principle of risk management [10]. For this reason, all aspects of health and safety and quality management are said to incorporate a degree of risk. Thus, they require a risk management process that helps to prioritize, evaluate, and handle a risk appropriately. As a result, it is appropriate to identify and understand the risks that are common in the working practices in the lab in order to manage them. The management system can manage these risks by establishing a risk management process that involves identifying, analyzing and evaluation, and eliminating [11]. Although it is not possible to identify and eliminate all risks, in reality the risk management process is practical and possible.

\section{Risk Identification}

Medicine and laboratory chemicals are risky operation, which require critical management and assessment. The management appoints a group of individuals in the lab and assigns them different roles that help to identify risks. More so, different professions and grades of staff are best placed to ensure the identification of risks, which in most cases are narrowed down by dividing them into different categories [11]. This depicts that the least and broadest possible range of risk in the laboratory operations are included. In the process of identification, lab incidents are investigated and established in order to analyze their risks and come up with possible additional measures to ensure they are not repeated or are avoided completely [12]. When proper identification and monitoring is utilized the likelihood is that loopholes that pose risk will decrease. More so, identifying possible risks that affect the staff and eliminating them may have positive effects on the staff as it will boost their morale.

\section{Risk Analysis and Evaluation}

After identification, analyzing and evaluation a potential risk in laboratory evaluation is a vital part of the quality control process. Through this process, the management is able to identify the severity and risk of the problem. By evaluating and analyzing the risk, the management is, therefore, able to use tools and establish procedure that will put a value on the specific risk, as well as, prioritize the risk before further action [13]. In most cases, the risk manager puts up a system in place where accidents and incidents are reported, however small. The data is then recorded, and the manager is able to see the full picture after careful evaluation.

\section{Risk elimination}

The main objective behind identifying and evaluation a potential risk in the laboratory is to eliminate or control the potential risk. It should be noteworthy that in most circumstances, it is possible to avoid a risk and even prevent is completely, but only is identified and analyzed. For instances, prior to the 1970s, the use of some chemical such as mercuric chloride was common, yet it was extremely harmful 
for the environment, and also poses health risks to the laboratory staff. After careful identification and evaluation, its use was subsequently stopped and it was replaced with another alternative. This efforts and actions indicate that it is possible to eliminate a risk and reduce possible impacts from happening. However, there are numerous ways of eliminating risks, and, in most cases, it may require guidance from an expert. In other cases, regulations are issued by the government or professional bodies, and the risk manager should in turn ensure they are implemented.

\section{Role of Technical Quality Control}

In addition to quality management system, Quality Control (QC) plays a major role as it ensures consistent, precise and accurate laboratory test results. This program checks that the work process is functioning properly by performing procedures that recognize and eliminate errors in the laboratory. The law requires that every laboratory to have an ongoing, carefully performed and well documented quality control program in place. In fact, quality control has all been an important component for laboratory accreditation and is ingrained in histotechnologists as a daily routine [14]. Thus, laboratory director must be certain that the quality control guidelines are followed in order to comply with the law.

The quality control program is designed to monitor all components of laboratory procedures, taking account of specimen collection and processing, test methodology, and reporting of test results. Furthermore, this program is not only used to monitor test results, but it also monitors equipments, reagents used in testing, personnel and supplied [15]. For this reason, this program plays a major role as discourages the release of incorrect results, and emphasizing on desired level of quality. However, it should be noted that quality assurance should not eliminate the need for a quality control program because this program should be continuous. A good quality program should include:

- A well-developed laboratory procedure manual that takes account of all pertinent information for every test.

- An equipment record that contains the exact record on all equipment used to test samples

- Calibrating instruments is mandatory, and therefore, the calibrating procedure should be performed on all lab equipments as per the manufacturer's instructions.

- Checking and recording daily temperature.

- Ensure that all equipment are well maintained as recommended by the manufacturer.

- Double checking the test methods before reporting making any report.

\section{Internal and external quality control}

Quality control is comprised of internal and external quality testing. These testing procedures are usually mandated by the regulatory agencies and government regulations, which also provide standards, guidelines and regulations [16]. Internal quality testing is usually in house, because the procedure occurs within the laboratory. This form of testing ensures a continuous monitoring procedure in the operation of the day to day produced data to decide whether the data is reliable enough to be released. The primary aim of the internal quality program is to monitor biased data with the help of control samples. According to [2], internal quality control is applied in manual techniques such as immunocytochemistry analyses and special staining procedures of tissue sections in cytopathology laboratories and histopathology. Discrepancies in results are investigated, where major discrepancies may require repeat test, possibly with request for another specimen [17].

\section{Role of Quality Control in Histology Laboratories}

External quality control involves the assistance from other laboratories, as well as, the participation in national and/or international inter-laboratory sample and data exchange programs (Vernon). The assessment of data from many laboratories and comparison of results with those of various sites ensures a continuity of testing over geography. Data from all participating laboratories are collated and presented as a report to show performance of a laboratory in two, which involves; comparing the lab with peer labs, and checking whether the performance is below the minimum standards. Although the external testing is done retrospectively and periodically, many countries require the participation of laboratories in external quality program in order to promote consistent and accurate test results [18]. Both internal and external testing plays a major role as they monitor effectiveness of quality management system, document occurrences, and identify opportunities for an improve performance.

\section{Continuous quality improvement}

A continuous quality improvement (CQI) program, also known as a quality improvement, would also improve the provision of services by focusing on a future state or a result from the current state [19]. Thus, the program aims at identifying any problem regarding the quality of services provided and allows the improvement of those services. When a process that requires improvement is identified, a procedure is put in place to measure the output, and suggest changes to be implemented. The program plays a major role as it aims at preventing future failures by helping to set future goals. It is important for manager to realize that many abnormalities and errors are not only caused by the laboratory staff but they may arise due to the system process.

In addition, quality control in histology labs enhances their competitiveness and makes them better workplace environments. When a laboratory has a continuous quality improvement program in place, it becomes more competitive and a better place for employment. The success of the CQI program is measurable, and it is usually determined through effective evaluation. Evaluation helps to review the program in order to determine the extent in which the process have improved, or determine the changes that need to be implemented [3]. For instance, a thorough and regular audit should be mapped to identify any problem. The feedback of users is also another effective way of analysing the success of the process because they provide useful information concerning the current quality of service. In case, an organization receives any criticism, it should be able to realize some form of abnormality, errors or failures in the system and identify. This will help the organization to arrive at the corrective and preventive measure that will remove the root cause.

In addition, quality control in histology laboratories reduces the possibility for the occurrence of hazards and accidents. It is well acknowledged that almost every other material used in histology labs is toxic and hazardous to any user especially when handled inappropriately. Of particular note is the fact that quality control does not only concentrate on the nature of products used, but also the manner in which they are handled or even stored. Proper handling 
Page 4 of 5

would ensure that the toxic materials do not pose any risk to the individuals, while still maintaining their integrity. These hazards arise from the wide range of chemical used in the histological laboratories pose potential risks. As a result, all laboratories are required to implement safety measure and regulation that ensure that all laboratory workers are protected. These measures may involve instructions in handling dangerous chemical substances, the need for labeling chemical containers, the adherence to expiry date as instructed by the manufacturer, and the storage conditions of chemicals and dangerous reagents [20]. These measures function to minimize risks occurring within the laboratories due to mishandling of chemical substances.

\section{Quality Assurance}

All laboratories should recognize the relevance of quality of an effective quality insurance program. Much stress has been made on the need for quality and consistency, as it is the first step towards getting every program and procedure right. Therefore, like all other programs, the quality assurance program means more than just adopting a protocol or obtaining an accreditation. The role of this program is to assure confidence in the quality and show a completeness of integrity of the data. It includes a systematic action that gives adequate confidence that a product or service has met the requirement that satisfies the level of quality [21]. Quality assurance may encompass continued education of staff, training, double screening, and review of laboratory procedures.

The management and laboratory staff should realize that effective quality management will only result by recognizing various components of quality assurance. These components may involve procedure required in report writing, audits and review of testimonies among others [22]. This should well pointed and illustrated through work notes that adequately explain what examiners did in order to reach the conclusive result. Furthermore, the management should recognize that they play a vital role in the quality assurance, because this can not only be achieved by the laboratory staff. The quality assurance program can be divided into three phases that include; the pre-analytical phase, analytical phase and the post-analytical phase.

\section{Pre-analytical phase}

Experts holds that $40 \%$ of laboratory errors take place in the preanalytical phase, while another $40 \%$ take place in the post-analytical phase, and $20 \%$ in the analytical phase. The pre-analytical phase, also known as the examination process, involve patient preparation, collecting samples, labeling them, transporting the samples for testing, and preparing the samples. Proper care is required in this process in proper collection and labeling, as well as, the use of clean devices for using transportation to avoid major mistakes.

\section{Analytical phase}

The analytical phase, which is better known as the examination process, includes events that include the actual performance of the lab procedures. This may involve the need of quality control that result to accuracy and precision of tests performed. This phase plays a key role in assuring and continually improving the quality of medical care. Most experts urge that undergraduate medical students require formal involvement in the analytical phase [23].

\section{Post-analytical phase}

This phase, also known as the post-examination process, involves clarity, accuracy and thoroughness of handwritten reports. The main events that take place in the post-analytical phase include transcription of patient's data onto the final report, typing and distribution of the report [24]. Much emphasis is made on the on the importance of calculations and double checking of the calculations. Tallying of the deferential count and lipid fractions before signing out of the report is a must. This is because; a small error in the report may cost the laboratory of its confidence with the patient [21]. A laboratory may have used all the required technology and reagents, but a slight mistake in this phase has the potential to make all the hard work redundant.

\section{Conclusion}

Histology laboratories must provide high quality services by enforcing a technical quality control system. This can only be achieved by provided accurate, relevant, precise and comprehensive data, which is applied to medical management of patients. Any misdiagnosis or errors on reports can lead to massive impacts on the quality of service and health consequences to patients. Thus, laboratories must follow quality rules and standards that are already established. More so, the goal of quality management in laboratories should be to gain continuous improvement, satisfaction of product and services to consumers, and guarantee quality laboratory results.

\section{References}

1. Mercer R (2012) How to Land a Top-Paying Histology Technicians Job: Your Complete Guide to Opportunities, Resumes and Cover Letters, Interviews, Salaries, Promotions, What to Expect from Recruiters and More. Yorkshire: Emereo Pty Limited Careers in Focus.

2. Buesa RJ (2005) Quantifying quality: a review and a scale proposal. J Histotechnol 28: 89 - 97.

3. Buesa RJ (2007) Histology: a unique area of the medical laboratory. Ann DiagnPathol 11: 137-141.

4. Gass J (2012) Needs Analysis and Situational Analysis: Designing an ESP Curriculum for Thai Nurses. English for Specific Purposes World 12: 37-54.

5. Iyengar JN (2009) Quality control in the histopathology laboratory: an overview with stress on the need for a structured national external quality assessment scheme. Indian J PatholMicrobiol 52: 1-5.

6. Berte LM (2007) Laboratory quality management: a roadmap. Clin Lab Med 27: 771-790, vi.

7. Sharif MQ, Mushtaq S, Mamoon N, Jamal S, Luqman M (2007) Clinician's responsibility in pre-analytical quality assurance of histopathology. Pak J Med Sci 23: 720-723.

8. Sergi C, Mikuz G (2008) External quality assurance as a revalidation method for pathologists in pediatric histopathology: Comparison of four international programs. BMC ClinPathol 8: 11.

9. Ortiz E, Spalding L, Andreasen JB (2011) Cliffs Notes FTCE: Elementary Education K6. Florida: Houghton Mifflin Harcourt.

10. Raab SS, Nakhleh RE, Ruby SG (2005) Patient safety in anatomic pathology: measuring discrepancy frequencies and causes. Arch Pathol Lab Med 129: 459-466.

11. Dapson JC, Dapson RW (2005) Hazardous materials in the histopathology laboratory: regulations, risks, handling and disposal. (4th edn), Battle Creek, Anatech Ltd.

12. Bui MM, Smith P, Agresta SV, Cheong D, Letson GD (2008) Practical issues of intraoperative frozen section diagnosis of bone and soft tissue lesions. Cancer Control 15: 7-12. 
Citation: Mohammedsaleh ZM (2014) The Role of Technical Quality Control in Histology Laboratories. J Cytol Histol 5: 264. doi: 10.4172/2157-7099.1000264

Page 5 of 5

13. Buesa RJ (2007) “Examining costs” (costs of histology procedures). Adv Med Lab Prof.

14. Ejilemele AA, Ojule AC (2005) Knowledge, attitude and practice of aspects of laboratory safety in Pathology Laboratories at the University of Port Harcourt Teaching Hospital, Nigeria. Niger J ClinPract 8: 102-106.

15. Hollensead SC, Lockwood WB, Elin RJ (2004) Errors in pathology and laboratory medicine: consequences and prevention. J SurgOncol 88: 161-181.

16. Buesa RJ (2006) Removing the stumbling blocks. Advance Med Lab Profess 15: 76.

17. Titford M (2006) A short history of histopathology technique. J Histotechnol.

18. Valenstein PN, Souers R, Wilkinson DS; College of American Pathologists (2005) Staffing benchmarks for clinical laboratories: a College of American Pathologists Q-probes study of staffing at 151 institutions. Arch Pathol Lab Med 129: 467-473.
19. Steward CA, Thompson NN (2006) ASCP 2005 wage and vacancy survey of medical laboratories. Lab Med 37: 465-469.

20. Vernon SE (2005) Continuous throughput rapid tissue processing revolutionizes histopathology work flow. Path Lab Med 36: 300-302.

21. Rolls G (2005) An evaluation of xylene-free processing of tissues from the central nervous system using Peloris dual retort rapid tissue processor. Mount Waverley, Victoria, Australia; Vision Bio Systems Ltd.

22. Buesa RJ (2004) Letter to the editor [about the "special report" from the NSH productivity task force]. J Histotechnol.

23. Buesa RJ (2006) Removing the stumbling blocks. Advance Med Lab Profess 15: 76

24. LaFriniere M, Lewis S, Sheppard B, Carson F (2004) A report from the National Society of Histotechnology productivity task force. J Histotechnol 27: 293-295. 